\title{
Interacção de Iões Tiocianato e Perclorato em Meio Ácido
}

$O$ perclorato de sódio é utilizado frequentemente para a manutenção da força iónica em soluções destinadas ao estudo cinético. No entanto, em certas condições, pode dar origem a reacções interferentes.

Procuramos estabelecer as condições em que se dá a reacção redox entre perclorato e tiocianato de sódio, em meio ácido, que pode conduzir, inclusivamente, à decomposição dos reagentes.

\section{Introdução}

$\mathrm{O}$ uso do sistema $\mathrm{NaClO}_{4} / \mathrm{HClO}_{4}$ é frequente no estudo cinético de reacções envolvendo espécies iónicas, a fim de controlar a força iónica e, dentro de certos limites, a acidez do meio.

Ao estudarmos a reacção da anionação entre o perclorato $\alpha \beta$ anti-aquo [1,9 bis(2'piridilo)2,5,8triazanonano]cobalto (III) e o ião tiocianato [1], verificamos que, ao utilizarmos o tampão atrás descrito, podíamos obter reacções secundárias que alteram por completo os iões reagentes, provocando reacções secundárias que tivemos de evitar.

Através de um estudo baseado na identificação dos produtos obtidos, procuramos interpretar as diferentes reacções que podem ocorrer.

\section{Parte experimental}

Utilizamos reagentes de grau analítico, que não submetemos a qualquer tratamento posterior, excepto a secagem em estufa a $110^{\circ} \mathrm{C}$ do perclorato e tiocianato de sódio, durante 48 horas.

A concentração do ácido perclórico e das soluções de perclorato de sódio foi determinada por titulação potenciométrica. Uma descrição sumária das experiências realizadas, encontra-se na Tabela I.

Estas observações experimentais suscitam algumas questões:

i) Poderá a cor rosa observada nas experiências 1, 2, 3, 5, 6 e 7 ser explicada pela formação de polímeros (NCS)?

ii) A solução 4 é incolor. Não haverá reacção nestas condições?

iii) Qual a(s) espécie(s) responsável pela solução amareloesverdeada nas experiências 5 e 6 ?

iv) $\mathrm{O}$ precipitado amarelo-pálido obtido nas experiências 5 e 6 poderá ser enxofre?

A fim de respondermos à questão i) traçamos os espectros de VIS-UV entre $280-520 \mathrm{~nm}$ que se apresentam na Figura 1,

TABELA I

Sumário das experiências realizadas

\begin{tabular}{|c|c|c|c|}
\hline EXPERIÊNCIA & {$\left[\mathrm{HClO}_{4}\right]$} & [NaNCS] & OBSERVAÇÕES EXPERIMENTAIS \\
\hline 1 & $1.0 \mathrm{M}$ & $0.5 \mathrm{M}$ & Solução rosa pálido (formação instantânea de cor) \\
\hline 2 & $0.05 \mathrm{M}$ & $0.5 \mathrm{M}$ & Solução rosa pálido (formação instantânea de cor) \\
\hline 3 & $1.0 \mathrm{M}$ & $0.075 \mathrm{M}$ & Solução rosa pálido (formação instantânea de cor) \\
\hline 4 & $0.05 \mathrm{M}$ & $0.075 \mathrm{M}$ & Solução incolor \\
\hline 5 & $\begin{array}{c}60 \%(\mathrm{w}-\mathrm{w}) \\
2 \text { partes }\end{array}$ & $\begin{array}{c}\text { Satur. } \\
1 \text { parte }\end{array}$ & $\begin{array}{l}\text { Sol. rosa intenso (form. inst. cor-dura pouco tempo) } \rightarrow \text { Sol. } \\
\text { amarelo-esverdeada (minutos) } \rightarrow \text { pp. amarelo pálido }+ \text { gases } \\
\text { abundantes }\end{array}$ \\
\hline 6 & $\begin{array}{c}60 \%(\mathrm{w}-\mathrm{w}) \\
\text { excesso }\end{array}$ & cristais & $\begin{array}{l}\text { Sol. rosa intenso em torno dos cristais (form. inst. cor, dura } \\
\text { segundos) } \rightarrow \text { sol. amarelo-esverdeada (minutos) } \rightarrow \text { pp. amarelo } \\
\text { pálido + gases abund. }\end{array}$ \\
\hline 7 & $\begin{array}{c}60 \%(\mathrm{w}-\mathrm{w}) \\
\text { só cobrir } \\
\text { cristais }\end{array}$ & cristais & $\begin{array}{l}\text { Sol. laranja int. (form. inst., dura segundos) } \rightarrow \text { sol. sobrenadante } \\
\text { verm. + sol. leitosa inferior, dura segundos } \rightarrow \text { sol. amar. }+ \text { pp. } \\
\text { verm. escuro (abund.) + susp. amar-laranja } \rightarrow \text { form. gases (lenta) }\end{array}$ \\
\hline
\end{tabular}

a Centro de Electroquímica e Cinética da Universidade de Lisboa no Instituto Rocha Cabral, Departamento de Química da Faculdade de Ciências de Lisboa 
para os vários intervenientes na reacção, utilizando água tridestilada como branco. Se considerarmos que a extensão da reacção entre o $\mathrm{HClO}_{4}$ e o NaNCS só se dá em pequena escala nas condições experimentais, podemos interpretar os resultados como a soma do espectro do ião tiocianato com o espectro do polímero.

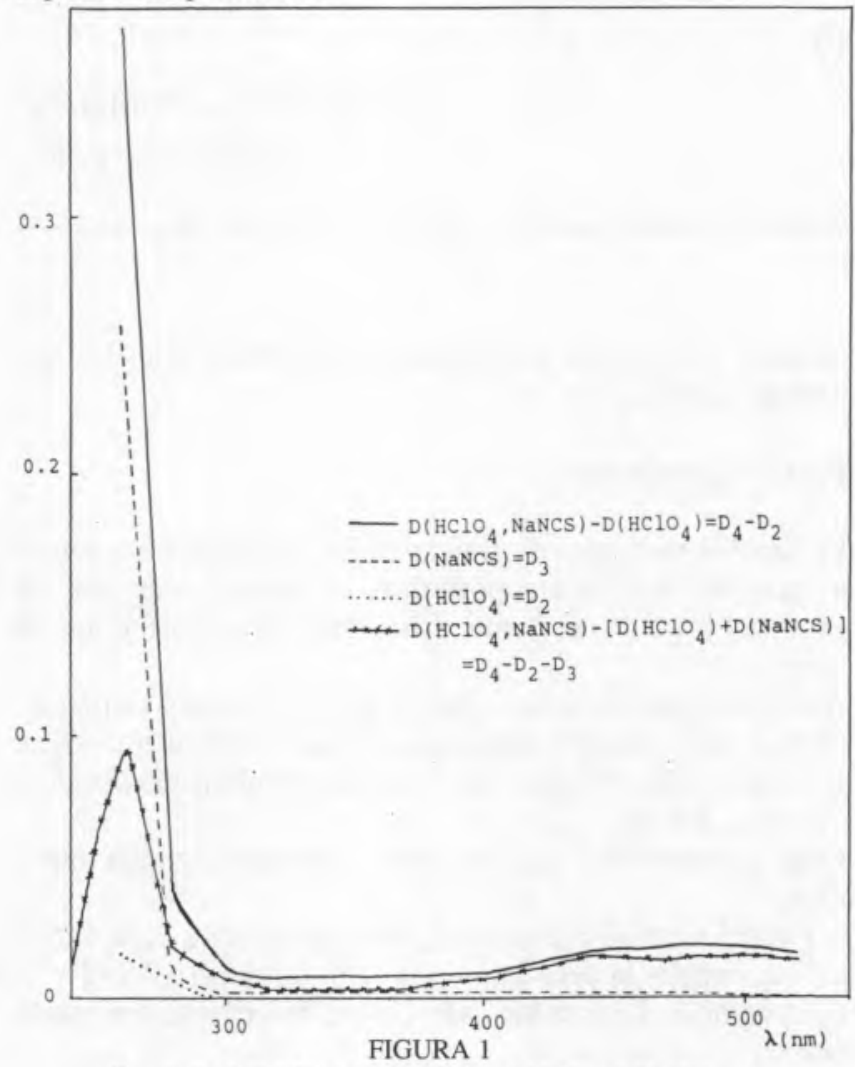

Espectros dos vários componentes da mistura reaccional

A resposta à questão ii) não pode ser dada inequivocamente. Para dar resposta à questão iii) realizaram-se testes para identificação do $\mathrm{H}_{2} \mathrm{~S}$ e do $\mathrm{SO}_{2}$ [2] que se baseiam nas seguintes reacções:

$$
\begin{aligned}
& \mathrm{Pb}\left(\mathrm{CH}_{3} \mathrm{COO}\right)_{2}+\mathrm{H}_{2} \mathrm{~S} \rightleftarrows \mathrm{PbS}+2 \mathrm{HCH}_{3} \mathrm{COO}
\end{aligned}
$$

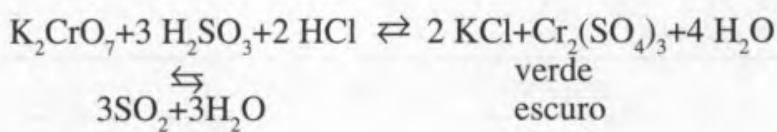

Os resultados destes testes encontram-se na Tabela II. O precipitado obtido nas experiências 5 e 6 é enxofre, como se verificou através das suas propriedades físicas (fusão e sublimação).

\section{Interpretação de resultados}

Este conjunto de experiências permite-nos tirar algumas conclusões interessantes.

O tiocianogénio (NCS) ${ }_{2}$ pode formar-se por oxidação do ião tiocianato com dióxido de manganês [3]:

$$
\mathrm{MnO}_{2}+2 \mathrm{NCS}+4 \mathrm{H}^{+} \rightleftarrows(\mathrm{NCS})_{2}+\mathrm{Mn}^{2+}+2 \mathrm{H}_{2} \mathrm{O}
$$

Este esquema químico pode ser decomposto nas seguintes reacções:

$$
\begin{array}{ll}
2 \mathrm{NCS}^{-} \rightleftarrows(\mathrm{NCS})_{2}+2 \mathrm{e}^{-} & \mathrm{E}^{\circ} \text { red }=0.77 \mathrm{~V} \\
\mathrm{MnO}_{2}+4 \mathrm{H}^{+}+2 \mathrm{e}^{-} \rightleftarrows \mathrm{Mn}^{2+}+2 \mathrm{H}_{2} \mathrm{O} & \mathrm{E}^{\circ} \text { red }=1.23 \mathrm{~V}
\end{array}
$$

$\mathrm{O}$ ião $\mathrm{ClO}_{4}^{-}$faz parte do par redox $\mathrm{ClO}_{4}^{-} / \mathrm{ClO}_{3}^{-}\left(\mathrm{E}^{\circ}\right.$ red=1.19 V) [4], semelhante ao do par redox $\mathrm{MnO}_{2} / \mathrm{Mn}^{2+}\left(\mathrm{E}^{\circ}\right.$ red $=1.21$ V) o que nos leva a considerar que o primeiro par pode também oxidar o tiocianato a tiocianogénio:

$$
\mathrm{ClO}_{4}^{-}+2 \mathrm{NCS}^{-}+2 \mathrm{H}^{+} \rightleftarrows(\mathrm{NCS})_{2}+\mathrm{ClO}_{3}^{-}+\mathrm{H}_{2} \mathrm{O}
$$

O facto da reacção ser energeticamente favorável não implica que ela possa ocorrer a uma velocidade mensurável, isto é, pode ser muito lenta, bastando para tal, que a energia de activação do processo seja muito elevada.

GOULD [5] afirma que a redução do perclorato a clorato, a frio, é extremamente lenta em soluções diluídas de $\mathrm{HClO}_{4}$ com redutores como o iodeto de potássio e o Fe (II). $\mathrm{O}$ mesmo autor afirma que o tiacianogénio só é estável a baixas temperaturas e que à temperatura ambiente polimeriza (NCS) precipitando na forma de uma massa vermelha, quando $\mathrm{x}$ é suficientemente grande.

$\mathrm{O}$ aumento de absorção na região dos $400-520 \mathrm{~nm}$ pode ser consistente com a transmissão de uma cor rosa, significando que se formam pequenas quantidades de (NCS) $\mathrm{em}$ que a absortividade molar dos polímeros é grande. De qualquer modo, os resultados experimentais indicam que $\varepsilon_{(\mathrm{NCS})_{\mathrm{x}}}>\mathrm{X} . \varepsilon_{\mathrm{NCS}}-$ para qualquer comprimento de onda, pois de outro modo não se poderia registar o aumento de absorvância verificado quando se passa da curva $D_{3}$ vs $\lambda$ para $D_{4^{-}}$ $\mathrm{D}_{2}$ vs $\lambda$.

$\mathrm{O}^{2}$ seguinte conjunto de reacções pode explicar o conjunto de testes negativos do dicromato:

$$
\begin{gathered}
\mathrm{K}_{2} \mathrm{Cr}_{2} \mathrm{O}_{7}+3 \mathrm{H}_{2} \mathrm{~S}+8 \mathrm{HCl} \rightleftarrows 2 \mathrm{KCl}+2\left[\mathrm{Cr}\left(\mathrm{H}_{2} \mathrm{O}\right)_{3} \mathrm{Cl}_{3}\right]+\mathrm{H}_{2} \mathrm{O}+\mathrm{S} \\
\mathrm{K}_{2} \mathrm{Cr}_{2} \mathrm{O}_{7}+6 \mathrm{ClO}_{2}+8 \mathrm{HCl}+5 \mathrm{H}_{2} \mathrm{O} \rightleftarrows 2 \mathrm{KCl}+2\left[\mathrm{Cr}_{2}\left(\mathrm{H}_{2} \mathrm{O}\right)_{3} \mathrm{C}_{3}\right]+6 \mathrm{HClO}_{3} \\
\mathrm{~K}_{2} \mathrm{Cr}_{2} \mathrm{O}_{7}+3 \mathrm{Cl}_{2}+4 \mathrm{HCl}+5 \mathrm{H}_{2} \mathrm{O} \rightleftarrows 2 \mathrm{KCl}+2\left[\mathrm{Cr}_{2}\left(\mathrm{H}_{2} \mathrm{O}\right)_{3} \mathrm{Cl}_{3}\right]+2 \mathrm{HClO}_{3} \\
\left.\left[\mathrm{Cr}_{2} \mathrm{H}_{2} \mathrm{O}\right)_{3} \mathrm{Cl}_{3}\right]+3 \mathrm{H}_{2} \mathrm{O} \rightleftarrows\left[\mathrm{Cr}\left(\mathrm{H}_{2} \mathrm{O}\right)_{6}\right]^{3+}+3 \mathrm{Cl}^{-}
\end{gathered}
$$

Assim, os testes negativos servem para confirmar o resultado positivo do teste do acetato de chumbo, mas não servem para identificar inequivocamente $\mathrm{ClO}_{2}$ e/ou $\mathrm{Cl}_{2}$.

As soluções 5 e 6 apresentavam, a dada altura, um cheiro a lexívia, o que pode indicar que durante a reacção se forma ácido hipocloroso e/ou cloro. A solução apresenta também uma cor esverdeada, que poderia resultar da dissolução do cloro formado.

$\mathrm{O}$ malogro da detecção do $\mathrm{SO}_{2}$ pode ter sido devido à interferência da reacção com $\mathrm{H}_{2} \mathrm{~S}$ e/ou $\mathrm{ClO}_{2}$ ou $\mathrm{Cl}_{2}$.

$\mathrm{O}$ teste do permanganato, que permite também detectar o dióxido de enxofre não se realizou devido a que, em meio ácido, como o teste requer, ocorrem interferências com o $\mathrm{H}_{2} \mathrm{~S}$ :

$$
\text { ou } \begin{gathered}
2 \mathrm{MnO}_{4}+5 \mathrm{H}_{2} \mathrm{~S}+6 \mathrm{H}^{+} \\
2 \mathrm{MnO}_{4}^{-}+5 \mathrm{H}_{2} \mathrm{~S}+5 \mathrm{O}_{2}+6 \mathrm{H}^{+} \rightleftarrows 5 \mathrm{Mn}^{2+}+8 \mathrm{H}_{2} \mathrm{O} \\
\rightleftarrows 2 \mathrm{Mn}^{2+}+8 \mathrm{H}_{2} \mathrm{O}
\end{gathered}
$$

$\mathrm{Na}$ reacção entre o $\mathrm{HClO}_{4}$ e o NaNCS podemos ainda considerar que se forma $\mathrm{HSO}_{3}$ e $\mathrm{CN}^{-}$, mas em meio ácido devem ocorrer ainda, as reacções:

$$
\begin{aligned}
\mathrm{SO}_{3}+2 \mathrm{H}^{+} \rightleftarrows \mathrm{H}_{2} \mathrm{SO}_{3} \rightleftarrows & \underset{\mathrm{SO}_{2}(\mathrm{aq})+\mathrm{H}_{2} \mathrm{O}}{\rightleftarrows} \\
& \stackrel{\mathrm{SO}_{2}(\mathrm{~g})}{\mathrm{CN}+\mathrm{H}^{+} \rightleftarrows \mathrm{HCN}(\mathrm{aq})} \rightleftarrows \mathrm{HCN}(\mathrm{g})^{\rightleftarrows}
\end{aligned}
$$


Mas, as quantidades de $\mathrm{SO}_{2}(\mathrm{~g})$ e $\mathrm{HCN}(\mathrm{g})$ que se podem libertar devem ser mínimas, pois na mistura reaccional pode ocorrer a seguinte reacção:

$$
2 \mathrm{H}_{2} \mathrm{SO}_{3}+2 \mathrm{HCN}+\mathrm{H}_{2} \mathrm{O} \rightleftarrows\left(\mathrm{NH}_{4}\right)_{2} \mathrm{~S}_{2} \mathrm{O}_{3}+2 \mathrm{CO}_{2}(\mathrm{~g})
$$

que consome os ácidos formados, impedindo a libertação de gases.

Esta reacção é relativamente rápida quando comparada com as reacções que produzem $\mathrm{H}_{2} \mathrm{SO}_{3}$ e $\mathrm{HCN}$.

$\mathrm{O}$ precipitado abundante, formado no decurso da experiência 7 deve ser o polímero (NCS).

Algum tempo depois, verifica-se o aparecimento de uma suspensão amarelo-alaranjada. Esta alteração deve-se, provavelmente, ao facto deste polímero ser atacado por espécies oxidantes que o vão desagregando e formando enxofre na sua superfície.

A formação do precipitado de (NCS), na experiência 7 e a formação instantânea da cor rosa intensa nas experiências 5 e 6 parece indicar que o primeiro passo das reacções entre o $\mathrm{HClO}_{4}$ e o NaNCS deve ser a oxidação do tiocianato a tiacianogénio:

$$
\mathrm{ClO}_{4}+2 \mathrm{NCS}^{-}+2 \mathrm{H}^{+} \rightleftarrows \mathrm{ClO}_{3}^{-}+(\mathrm{NCS})_{2}+\mathrm{H}_{2} \mathrm{O}
$$

e que nas experiências 5 e 6 não se verificou a formação do precipitado vermelho-escuro porque deveriam existir condições para que o (NCS), reagisse mais rapidamente por uma via distinta da reacção de polimerização.

$\mathrm{O}$ conjunto de todos os resultados experimentais indica que

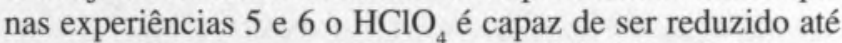
$\mathrm{Cl}_{2}$ (e possivelmente até $\mathrm{Cl}^{-}$) e que o NCS é passível de ser oxidado até à formação de $\mathrm{S}, \mathrm{H}_{2} \mathrm{~S}$ e $\mathrm{NH}_{4}^{+}$(e possivelmente $\mathrm{CO}_{2}$ ).

A seguinte combinação de "semi-reacções" poderia explicar grosseiramente o que se passa nas experiências 5 e 6 :

$$
\begin{aligned}
& \mathrm{ClO}_{4}+8 \mathrm{H}^{+}+8 \mathrm{e} \rightleftarrows \mathrm{Cl}+4 \mathrm{H}_{2} \mathrm{O} \\
& 2 \mathrm{NCS}^{-}+6 \mathrm{H}_{2} \mathrm{O} \rightleftarrows 2 \mathrm{HSO}_{3}+2 \mathrm{CN}^{-}+10 \mathrm{H}^{+}+8 \mathrm{e} \\
& 2 \mathrm{HSO}_{3}+12 \mathrm{H}^{+}+10 \mathrm{e} \rightleftarrows \mathrm{S}+\mathrm{H}_{2} \mathrm{~S}+6 \mathrm{H}_{2} \mathrm{O} \\
& 2 \mathrm{CN}^{-}+4 \mathrm{H}_{2} \mathrm{O} \rightleftarrows 2 \mathrm{CO}_{2}+2 \mathrm{NH}_{4}^{+}+4 \mathrm{e} \\
& 3 \mathrm{H}_{2} \mathrm{O} \rightleftarrows 3 / 2 \mathrm{O}_{2}+6 \mathrm{H}^{+}+6 \mathrm{e}
\end{aligned}
$$

$\mathrm{ClO}_{4}+2 \mathrm{NCS}+4 \mathrm{H}^{+}+3 \mathrm{H}_{2} \mathrm{O} \rightleftarrows \mathrm{S}+\mathrm{H}_{2} \mathrm{O}+3 / 2 \mathrm{O}_{2}+2 \mathrm{CO}_{2}+$ $+2 \mathrm{NH}_{4}{ }^{+}+\mathrm{Cl}^{-}$

À excepção da reacção (e) todas as outras são susceptíveis de serem decompostas em processos mais simples:

Reacção (a)

$$
\begin{aligned}
& \mathrm{ClO}_{4}^{-}+2 \mathrm{H}^{+}+2 \mathrm{e} \rightleftarrows \mathrm{ClO}_{3}^{-}+\mathrm{H}_{2} \mathrm{O} \ldots \\
& \mathrm{ClO}_{3}+3 \mathrm{H}^{+}+2 \mathrm{e} \rightleftarrows \mathrm{HClO}_{2}+\mathrm{H}_{2} \mathrm{O} \\
& \mathrm{HClO}_{2}+2 \mathrm{H}^{+}+2 \mathrm{e} \rightleftarrows \mathrm{HClO}_{+} \mathrm{H}_{2} \mathrm{O}
\end{aligned}
$$

$\mathrm{HClO}+\mathrm{H}^{+}+1 \mathrm{e} \rightleftarrows 1 / 2 \mathrm{Cl}_{2}+\mathrm{H}_{2} \mathrm{O}$

$1 / 2 \mathrm{Cl}_{2}+1 \mathrm{e} \rightleftarrows \mathrm{Cl}^{-}$

$\mathrm{ClO}_{4}^{-}+8 \mathrm{H}^{+}+8 \mathrm{e} \rightleftarrows \mathrm{Cl}+4 \mathrm{H}_{2} \mathrm{O}$

\author{
Reacção (b) \\ $2 \mathrm{NCS}^{-} \rightleftarrows(\mathrm{NCS})_{2}+2 \mathrm{e}$ \\ $(\mathrm{NCS})_{2}+3 \mathrm{H}_{2} \mathrm{O} \rightleftarrows \mathrm{HSO}_{3}+\mathrm{SCN}-\mathrm{CN}+5 \mathrm{H}^{+}+4 \mathrm{e}$ \\ $\mathrm{SCN}-\mathrm{CN}+3 \mathrm{H}_{2} \mathrm{O} \rightleftarrows \mathrm{HSO}_{3}+(\mathrm{CN})_{2}+5 \mathrm{H}^{+}+4 \mathrm{e}$ \\ $(\mathrm{CN})_{2}+2 \mathrm{e} \rightleftarrows 2 \mathrm{CN}^{-}$

$2 \mathrm{NCS}^{-}+6 \mathrm{H}_{2} \mathrm{O} \rightleftarrows 2 \mathrm{HSO}_{3}^{-}+2 \mathrm{CN}^{-}+10 \mathrm{H}^{+}+8 \mathrm{e}$

Reacção (c)

$$
\begin{aligned}
& 2 \mathrm{HSO}_{3}+4 \mathrm{H}^{+}+4 \mathrm{e} \rightleftarrows \mathrm{S}_{2} \mathrm{O}_{3}{ }^{2-}+3 \mathrm{H}_{2} \mathrm{O} \\
& \mathrm{S}_{2} \mathrm{O}_{3}{ }^{2-}+\mathrm{H}^{+} \rightleftarrows \mathrm{HS}_{2} \mathrm{O}_{3}^{-} \rightleftarrows \mathrm{HSO}_{3}^{-}+\mathrm{S} \\
& \mathrm{HSO}_{3}{ }^{-}+5 \mathrm{H}^{+}+4 \mathrm{e} \rightleftarrows \mathrm{S}+3 \mathrm{H}_{2} \mathrm{O} \\
& \mathrm{S}+2 \mathrm{H}^{+}+2 \mathrm{e} \rightleftarrows \mathrm{H}_{2} \mathrm{~S}
\end{aligned}
$$$$
2 \mathrm{HSO}_{3}+12 \mathrm{H}^{+}+10 \mathrm{e} \rightleftarrows \mathrm{S}+\mathrm{H}_{2} \mathrm{~S}+6 \mathrm{H}_{2} \mathrm{O}
$$

Reacção (d)

$$
\begin{aligned}
& 2 \mathrm{CN}^{-} \rightleftarrows(\mathrm{CN})_{2}+2 \mathrm{e} \\
& (\mathrm{CN})_{2}+2 \mathrm{H}_{2} \mathrm{O} \rightleftarrows \mathrm{CN}_{2}+\mathrm{CO}_{2}+4 \mathrm{H}^{+}+2 \mathrm{e} \\
& \mathrm{CN}_{2}{ }^{-}+2 \mathrm{H}^{+}+\mathrm{H}_{2} \mathrm{O} \rightleftarrows\left(\mathrm{NH}_{2}\right)_{2} \mathrm{CO} \\
& \left(\mathrm{NH}_{2}\right)_{2} \mathrm{CO}+2 \mathrm{H}^{+}+2 \mathrm{H}^{+}+2 \mathrm{H}_{2} \mathrm{O} \rightleftarrows 2 \mathrm{NH}_{4}^{+}+\mathrm{CO}_{2}
\end{aligned}
$$

$2 \mathrm{CN}^{-}+4 \mathrm{H}_{2} \mathrm{O} \rightleftarrows 2 \mathrm{CO}_{2}+2 \mathrm{NH}_{4}^{+}+4 \mathrm{e}$

Note-se que as reacções (a) e (c) são conjuntos de reduções sequenciais e que a formação de enxofre pode ocorrer ciclicamente, através dos processos:

$$
\begin{aligned}
& 2 \mathrm{HSO}_{3}^{-} \rightleftarrows \mathrm{S}_{2} \mathrm{O}_{3}{ }^{2-}+\mathrm{H}_{2} \mathrm{O}_{+} \mathrm{O}_{2} \\
& 2 \mathrm{~S}_{2} \mathrm{O}_{3}{ }^{2-}+2 \mathrm{H}^{+} \rightleftarrows \mathrm{HS}_{2} \mathrm{O}_{3}+\mathrm{HSO}_{3}+\mathrm{S}
\end{aligned}
$$

onde a primeira destas reacções resulta da soma do processo (c-1) com o processo (e).

Agrupando os vários processos mais simples entre si pode obter-se o seguinte conjunto de reacções globais de oxi-redução:

$$
\begin{aligned}
& \mathrm{ClO}_{4}+2 \mathrm{NCS}^{-}+2 \mathrm{H}^{+} \rightleftarrows \mathrm{ClO}_{3}+(\mathrm{NCS})_{2}+\mathrm{H}_{2} \mathrm{O} \\
& (\mathrm{NCS})_{2}+\mathrm{ClO}_{3}+3 \mathrm{H}_{2} \mathrm{O} \rightleftarrows 2 \mathrm{HSO}_{3}+2 \mathrm{HCN}^{-}+2 \mathrm{H}^{+}+\mathrm{Cl}^{-} \\
& 2 \mathrm{HSO}_{3}^{-}+2 \mathrm{HCN}^{-} 2 \mathrm{H}^{+}+\mathrm{H}_{2} \mathrm{O} \rightleftarrows \mathrm{S}_{2} \mathrm{O}_{3}^{2-}+2 \mathrm{CO}_{2}+2 \mathrm{NH}_{4}^{+} \\
& \mathrm{S}_{2} \mathrm{O}_{3}{ }^{2-}+2 \mathrm{H}^{+} \rightleftarrows \mathrm{S}+\mathrm{H}_{2} \mathrm{~S}+3 / 2 \mathrm{O}_{2} \\
& \mathrm{ClO}_{4}+2 \mathrm{NCS}^{-}+4 \mathrm{H}^{+}+3 \mathrm{H}_{2} \mathrm{O} \rightleftarrows \mathrm{S}+\mathrm{H}_{2} \mathrm{~S}+2 \mathrm{CO}_{2}+3 / 2 \mathrm{O}_{2}+ \\
& +2 \mathrm{NH}_{4}^{+}+\mathrm{Cl}^{-}
\end{aligned}
$$

A descrição do processo químico que apresentamos serve para salientar a complexidade da reacção entre $\mathrm{o} \mathrm{HClO}_{4}$ e o NaNCS, fornecendo-nos uma ideia dos intermediários possíveis e dos produtos que previsivelmente se obtêm, para além dos que conseguimos identificar experimentalmente. Esta expli- 
cação poderá não ser completa, mas é aquela que nos parece mais credível, face aos resultados experimentais.

Testes mais ou menos selectivos poderiam ter sido realizados, mas implicariam o uso de grandes concentrações de reagentes, o que nos parece de interesse relativamente reduzido, visto afastarmo-nos muito das condições das experiências cinéticas. Constatamos, no entanto, que é extremamente importante a relação das concentrações de $\mathrm{HClO}_{4}$ e NaNCS. radas e/ou ocorrer a uma velocidade menor do que em meio ácido.

As experiências realizadas indicam que a oxidação do tiocianato a tiocianogénio pode ocorrer a concentrações superiores a $0.05 \mathrm{M}$ de ácido e $0.075 \mathrm{M}$ de tiocianato de sódio. Nestas condições, não se verifica qualquer formação de cor, o que mesmo em pequena extensão, reforça a afirmação de que o ácido perclórico, apesar de ser um oxidante forte para com-

TABELA II

Resultados dos testes de identificação

\begin{tabular}{|c|c|c|c|}
\hline \multirow{2}{*}{ EXPERIÊNCIA } & \multicolumn{2}{|c|}{ TESTE } & \multirow{2}{*}{ CONCLUSÕES } \\
\hline & ACETATO DE CHUMBO & DICROMATO DE POTÁSSIO & \\
\hline 5 & papel enegrece & $\begin{array}{c}\text { papel violeta } \\
\text { com auréola amarela }\end{array}$ & $\begin{array}{c}\mathrm{H}_{2} \mathrm{~S} \text { presente } \\
\mathrm{ClO}_{2} / \mathrm{Cl}_{2} \text { possíveis }\end{array}$ \\
\hline 6 & papel enegrece & $\begin{array}{c}\text { papel violeta } \\
\text { com auréola amarela }\end{array}$ & $\begin{array}{c}\mathrm{H}_{2} \mathrm{~S} \text { presente } \\
\mathrm{ClO}_{2} / \mathrm{Cl}_{2} \text { possíveis }\end{array}$ \\
\hline 7 & $\begin{array}{l}\text { papel não enegrece antes } \\
\text { da formação pp. verm. } \\
\text { papel enegrece lentamente } \\
\text { ao formar pp. verm. }\end{array}$ & $\begin{array}{l}\text { sem alteração antes da form. } \\
\text { pp. verm. } \\
\text { papel violeta c/auréola amarela } \\
\text { após formar pp. verm. }\end{array}$ & $\begin{array}{c}\mathrm{H}_{2} \mathrm{~S} / \mathrm{ClO}_{2} / \mathrm{Cl}_{2} / \mathrm{SO}_{2} \\
\text { ausentes } \\
\mathrm{H}_{2} \mathrm{~S} \text { presente } \\
\mathrm{ClO}_{2} / \mathrm{Cl}_{2} \text { possíveis }\end{array}$ \\
\hline
\end{tabular}

Por exemplo, nas experiências 5, 6 e 7 obtém-se uma cor rosa intensa em torno dos cristais, que atribuímos à formação de (NCS) e que nas experiências 1, 2 e 3 se forma apenas, uma cor rosa pálida; podemos presumir que há duas vias distintas para a obtenção daqueles polímeros:

i) Nas experiências 5 e 6 , onde a quantidade de $\mathrm{HClO}_{4}$ é bem maior do que a de NaNCS, dá-se a formação rápida de (NCS) ${ }_{2}$ que polimeriza a (NCS), mas o meio continua suficientemente ácido para que as reacções (g), (h) e (i) possam ocorrer, competindo com a reacção de polimerização;

ii) $\mathrm{Na}$ experiência 7, onde a quantidade de $\mathrm{HClO}_{4}$ é semelhante ou ligeiramente menor do que a quantidade de NaNCS, continua a dar-se uma formação inicial rápida de $(\mathrm{NCS})_{2}$ e $(\mathrm{NCS})_{x}$, mas como o ácido é praticamente todo utilizado, todas as reacções subsequentes podem ser alte- postos orgânicos em soluções aquosas diluídas, à temperatura ambiente reage muito lentamente com compostos inorgânicos $[5,6]$.

\section{Referências}

[1] HUMANES, M. Comunicação XXVI International Conference on Coordination Compounds, Porto, 1988

[2] KING, E.J. "Qualitative Analysis and Electrolytic Solutions", Oliver \& Boyd, Londres, 1962

[3] HUHEEY, J.E. "Inorganic Chemistry - Principles of structure and reactivity", $3^{a}$ ed., Harper \& Row, Nova Iorque, 1983

[4] HANDBOOK OF CHEMISTRY AND PHYSICS, $53^{a}$ edição, CRC Press, 1973

[5] GOULD, E.S. "Inorganic reactions and structure", Holt, Rineheart \& Winston, Nova Iorque, 1962

[6]MAHAN, B.H. "Química - um curso universitário", 2" edição, Edit. Blis, São Paulo, 1976
NIÓBIO, de Niobe, filha do rei mítico grego Tantalos (o nióbio foi descoberto conjuntamente com o tântalo); descoberto em 1801. Usa-se em reactores atómicos, motores de aviões a jacto e foguetões. Até 1950 foi conhecido como Columbio, de Columbia - o nome poético da América, onde o seu minério foi primeiro descoberto.

MOLIBDÉNIO, de molybdos, ou chumbo (o molibdénio foi encontrado inicialmente no que se pensou ser minério de chumbo; descoberto em 1778. Tem o quinto mais elevado ponto de fusão de entre os metais. É usado em filamentos, caldeiras e canos de fuzis. Só em 1959 foi inventado um recipiente, um cadinho com água arrefecida, para o fundir.

TECNÉCIO, de technetos ou artificial; produzido em 1937. Primeiro elemento produzido pelo homem, originalmente pelo bombardeamento atómico do molibdénio; encontrou-se, mais tarde, entre os produtos da fissão do urânio. 